\title{
REVISIÓN
}

\section{Efecto de las alteraciones lipídicas sobre la calidad del pescado procesado}

\author{
Por Santiago P. Aubourg \\ Instituto de Investigaciones Marinas (CSIC). C/ Eduardo Cabello, 6. 36208 - Vigo (España) \\ Correo electrónico: saubourg@iim.csic.es
}

\begin{abstract}
RESUMEN
Efecto de las alteraciones lipídicas sobre la calidad del pescado procesado.

Las especies marinas constituyen un grupo de alimentos fácilmente dañable a lo largo del procesamiento empleado para su consumo. Entre las distintas vías de alteración que pueden actuar sobre un alimento de origen marino, destaca la facilidad a la oxidación que experimenta la fracción lipídica, debido a su alta proporción en ácidos grasos poliinsaturados.

En el presente trabajo se hace una revisión de la incidencia que los cambios producidos en la fracción lipídica de una especie marina pueden tener sobre el estado de calidad en cada uno de los principales procesos tecnológicos empleados (congelación y estado congelado, estado refrigerado, cocción, enlatado, secado, salazón y ahumado). Asimismo, se analiza la aplicabilidad de los principales índices de calidad basados en los cambios de la fracción lipídica, al objeto de establecer el grado de calidad en cada tipo de producto de pescado.
\end{abstract}

PALABRAS-CLAVE: Alteración - Calidad - Lípido - Pescado Procesamiento - Revisión (artículo).

\section{SUMMARY}

Effect of lipid damages on processed fish quality.

Marine species constitute a food group recognised as being easily damaged during the technological processing. Between the - different damage pathways that can act on a marine food, a big attention has been given to the oxidation ability of lipids, as a result of supporting a high content on polyunsaturated fatty acids.

In the present work, the role of lipid changes of a marine species on the quality degree is reviewed over the most employed technological processes (freezing and frozen storage, chilled storage, cooking, canning, drying, salting and smoking). The use of the main quality lipid indices for assessing the quality degree changes in each process is also analysed.

KEY-WORDS: Alteration - Fish - Lipid - Processing - Quality Review (paper).

\section{INTRODUCCIÓN}

Las especies marinas representan un grupo de alimentos importante en la dieta humana por su aporte en constituyentes fundamentales como ami- noácidos y ácidos grasos esenciales, vitaminas liposolubles y minerales (Navarro, 1991; Simopoulos, 1997).

Los alimentos de origen marino se caracterizan y distinguen de los demás alimentos por su composición lipídica altamente insaturada (Ackman, 1989). Entre los ácidos grasos poliinsaturados (AGPI) son de destacar los esenciales (linoleico, linolénico y araquidónico), pero sobre todo los de la denominada serie $\omega-3$, entre los que los más característicos son el docosahexaenoico ( $\mathrm{ADH}, \mathrm{C}_{22: 6}$ ) y el eicosapentaenoico ( $\mathrm{AEP}, \mathrm{C}_{20: 5}$ ). Numerosos estudios dietéticos y nutricionales muestran a los AGPI de la serie $\omega-3$ como responsables de la acción positiva de los productos marinos frente a determinadas enfermedades (Kinsella, 1987; Linko y Hayakawa, 1996).

Sin embargo, lo que desde un punto de vista nutritivo puede ser una ventaja, no lo es desde un punto de vista tecnológico. Los productos marinos son altamente perecederos, entre otros motivos por su composición lipídica altamente insaturada. Así, una vez que la actividad microbiana se encuentra detenida, entran en juego otras vías de alteración como la acción de las enzimas endógenas, la oxidación no enzimática de lípidos y el pardeamiento no enzimático; como resultado, la fracción lipídica adquiere un papel relevante, debido fundamentalmente a la fácil alterabilidad de sus ácidos grasos poliinsaturados.

\section{ALTERACIONES LIPÍDICAS Y SU SIGNIFICADO}

Tres tipos de reacciones pueden provocar la alteración de los lípidos: hidrolíticas, oxidativas y de entrecruzamiento. De todas ellas, son las de oxidación las que han acaparado mayor atención por tener una mayor incidencia sobre la calidad y valor nutritivo de un producto (Frankel, 1991; Harris y Tall, 1994). En la Figura 1 se muestra un esquema básico de la reacción de oxidación de lípidos, que incluye las distintas etapas de que consta. Como resultado se produce una amplia gama de productos, la mayoría con funciones oxigenadas, que incluyen desde moléculas volátiles hasta compuestos poliméricos. 


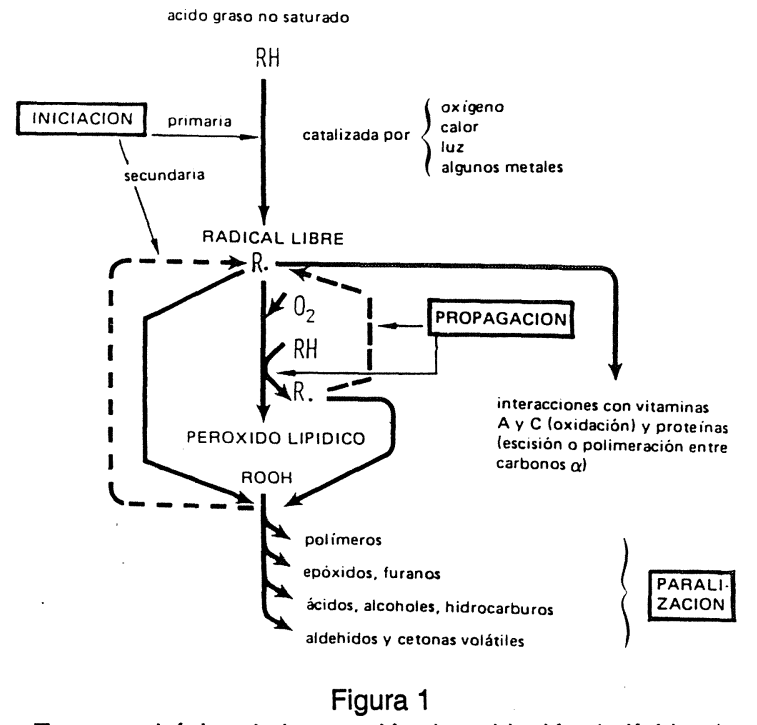

Esquema básico de la reacción de oxidación de lípidos *.

* Cheftel y Cheftel, 1976.

El carácter electrófilo de la mayoría de los productos de oxidación lipídica les lleva a reaccionar con constituyentes de los alimentos que sean portadores de funciones nucleófilas. Estas interacciones adquieren un papel muy importante en aquellos alimentos como los marinos, con alto contenido proteico y en aminoácidos esenciales como lisina y metionina.

Al igual que en las reacciones del tipo Maillard entre azúcares y aminoácidos, las interacciones entre lípidos oxidados y proteínas afectan al valor nutritivo y sensorial de los alimentos (Pokorný, 1981; Nielsen et al., 1985; Hidalgo et al., 1992). El valor nutritivo se puede ver afectado en los siguientes aspectos: a) Descenso del valor biológico de las proteínas (bloqueo de los grupos $\varepsilon$-amino en lisina; oxidación de los grupos sulfuro en metionina; cambios en otros aminoácidos); b) Cambios de digestibilidad (descenso de la velocidad de lipolisis por las lipasas; descenso de la velocidad y extensión de la proteolisis por las enzimas digestivas); c) Formación de sustancias tóxicas producidas por la autoxidación lipídica (hidroperóxidos lipídicos y aldehidos de bajo peso molecular).

La formación de compuestos de interacción entre lípidos oxidados y proteínas puede influir en el valor sensorial a nivel de: aroma (formación de nuevos compuestos aromáticos), color (reacción de pardeamiento) y textura (desnaturalización y entrecruzamiento proteicos).

\section{CAMBIOS LIPÍDICOS Y CALIDAD DEL PESCADO PROCESADO}

En los siguientes subapartados (3.1.-3.6.), se describirá la incidencia que la alteración de la frac- ción lipídica puede tener sobre la calidad del producto final durante el procesamiento de pescado, y en general de las especies marinas. Para ello se han escogido los principales tipos de procesos correspondientes a distintas estrategias seguidas para detener la alteración: acción del frío (congelación y refrigeración), calor (cocción, enlatado y ahumado) y control de la actividad de agua (salazón y secado).

\subsection{Congelación y estado congelado}

El objetivo principal de la congelación del pescado es impedir el crecimiento de microorganismos, retardar los fenómenos de degradación interna del pescado y conseguir que después de descongelado no se aprecien modificaciones respecto a las características que tenía en estado fresco. Sin embargo, numerosas experiencias demuestran que la oxidación e hidrólisis lipídicas, especialmente por vía enzimática, se siguen produciendo durante el almacenamiento en estado congelado.

Los efectos de alteración lipídica son importantes en las especies grasas, por lo que se han hecho numerosos estudios en torno al efecto catalítico de las enzimas del tipo lipoxigenasa (German et al., 1992; Mohri et al., 1992). Se ha observado que las especies grasas tienen un tiempo de vida útil inferior que las magras, llegándose a demostrar en la práctica que la formación de productos de oxidación es el factor limitante del tiempo de vida de una especie grasa en estado congelado (Madrid et al., 1994).

Las determinaciones de la hidrólisis y oxidación lipídicas han sido utilizadas con éxito en el seguimiento de la calidad de especies grasas (Verma et al., 1995; Karaçam y Boran, 1996). La medida de compuestos de interacción a través de sus propiedades fluorescentes ha proporcionado una buena correlación con el tiempo de almacenamiento y medidas de alteración lipídicas (ácidos grasos libres, AGL; índice de ácido tiobarbitúrico, i-ATB) (Aubourg et al., 1998).

En el caso del almacenamiento congelado de las especies magras, la alteración se ha centrado en los cambios de textura experimentados por el músculo. Así, la importancia de la participación lipídica en la alteración ha sido muy discutida, entendiendo que su incidencia era inferior a la de otras vías de alteración, como puede ser en el caso de las especies gádidas la presencia de formaldehido (FA) (Rehbein y Orlick, 1990); sin embargo, se ha reconocido la influencia de la fracción lipídica sobre la formación de FA y dimetilamina (Careche y Tejada, 1991; Joly et al., 1997). Además, tanto los AGL como los lípidos oxidados han sido propuestos como contribuyentes al fenómeno de desnaturalización proteica (Mackie, 1993; Sotelo et al., 1995). 
Las medidas de alteración lipídica durante la congelación de una especie magra se han centrado en la formación de AGL, llegándose a proponer como medida de calidad (de Koning y Mol, 1991), aunque la determinación de la oxidación también ha sido empleada (Pérez-Villarreal y Howgate, 1991; Fernández-Reiriz et al., 1995).

\subsection{Estado refrigerado}

Pescado refrigerado es el conservado en hielo desde su captura. Este es el sistema más utilizado a bordo, teniendo como excepción los casos en que la especie es procesada directamente a bordo (congelación en atuneros, salazón de bacalao, etc.). El factor más importante de cara a evitar la alteración del pescado como refrigerado es el mantenimiento de la temperatura a $0^{\circ} \mathrm{C}$. De los distintos mecanismos de alteración, el objetivo crucial y más importante es retardar la alteración microbiana hasta que el producto llega al consumidor. Por ello, las medidas más empleadas de seguimiento de la calidad han sido las relacionadas con la formación de bases volátiles (trimetilamina, principalmente) y aminas biógenas (histamina, principalmente). Las demás alteraciones pueden llevar a textura indeseable, cambios de color y desarrollo de rancidez; adquieren importancia en la medida que la alteración microbiana se encuentra paralizada.

En lo que respecta al papel de la fracción lipídica, se ha observado un buen seguimiento de la alteración a través de la medición de la hidrólisis (Nunes et al., 1992), llegándose incluso a proponer como índice de calidad alternativo a la determinación sensorial en pescado magro (Barassi et al., 1987). En el caso de la oxidación, ésta no parece ser un proceso dominante de alteración, aunque en casos de almacenamiento prolongado de especies grasas se pueden llegar a originar aromas y sabores desagradables, proporcionando un buen seguimiento de la alteración siempre y cuando se emplee más de un método de análisis (Ababouch et al., 1996; Olafsdóttir et al., 1997). Asimismo, se realizó la detección por fluorescencia de los compuestos de interacción entre productos de oxidación primaria y secundaria con otros constituyentes de pescado (Aubourg et al., 1997a), observándose una correlación satisfactoria de dicha medida con el tiempo de almacenamiento y el desarrollo de bases volátiles totales.

\subsection{Cocción}

Los tratamientos térmicos en general, representan el método más empleado para detener la acción microbiana y evitar cambios enzimáticos deteriorativos en muestras biológicas durante su al- macenamiento o posterior procesamiento. La cocción en concreto, se ha empleado con objeto de alargar la vida útil del producto destinado a posteriores procesamientos y como paso intermedio durante el enlatado (Pigott y Tucker, 1987; Mielche y Bertelsen, 1994).

Como resultado de la eliminación de la acción microbiana y la inactivación enzimática, la mayor atención de la alteración ha recaído sobre la fracción lipídica. Determinadas experiencias han demostrado ciertas alteraciones como lipolisis (Aubourg et al., 1989; Gallardo et al., 1989) y pequeñas perdidas de ADH y AEP (Tóth-Markus y Sass-Kiss, 1993). La detección de compuestos de interacción a través de sus propiedades fluorescentes demostró un incremento de la presencia de dichos compuestos como resultado del tratamiento (Aubourg et al., 1995a).

Sin embargo, son más numerosos los trabajos en que no se observan diferencias significativas antes y después de la cocción en los índices de calidad referentes a la fracción lipídica. Así, un amplio grupo de experiencias (Hearn et al., 1987; Suzuki et al., 1988; Gallardo et al., 1989) no detectaron diferencias en el contenido en AGPI. Tampoco se observó variación en los índices de peróxidos (IP) y de yodo (IY), a pesar de haberse realizado la experiencia en zonas del músculo de albacora con distinto contenido lipídico (Aubourg et al., 1989). Recientes aplicaciones de cocción a vacío («sous vide») indican asimismo, la retención de los ácidos grasos después del proceso (Schellekens, 1996).

\subsection{Enlatado}

Productos enlatados son los que se introducen en envases cerrados herméticamente y se someten a la acción del calor, con lo que se asegura la destrucción de todos los microorganismos, además de desnaturalizar las proteínas. En el caso de las especies marinas, la acción del calor y la presencia de determinados catalizadores pueden favorecer la oxidación de lípidos por vía no enzimática. La alteración de AGPI puede llevar a la formación de cantidades importantes de productos de oxidación primaria y secundaria, de manera que se produzca pardeamiento, compuestos fluorescentes, malos aromas y pérdidas de nutrientes esenciales. Como resultado, se concluye una importante relación entre los cambios lipídicos y la calidad del producto final.

La formación de AGL durante el enlatado de distintas zonas del músculo de albacora fue observada por Aubourg et al., (1990). Posteriormente, un estudio por RMN $-{ }^{13} \mathrm{C}$ demostró que la hidrólisis se producía preferentemente en la posición sn-2 de los triglicéridos frente a las posiciones sn-1 y sn-3, como es el caso del almacenamiento congelado (Medina et al., 1994). Se comprobó que el mecanismo y la 
extensión de la hidrólisis eran independientes del medio de cobertura (aceite o salmuera) empleado (Medina et al., 1995). Una comparación de distintas condiciones de esterilización demostró que tratamientos con menor duración pero mayor temperatura (a igual $F_{0}$ ) producían un mayor desarrollo de la hidrólisis (Aubourg et al., 1997b).

Se comprobó que la gran proporción de AGPI de los productos marinos se veía reducida en el producto resultante debido al contacto con el aceite vegetal empleado como medio de cobertura (Aubourg et al., 1990; Aubourg et al., 1995b); por ello algunos autores recomiendan el uso de aceites de cobertura con alto contenido en AGPI (Hale y Brown, 1983; GarcíaArias et al., 1991). Debido al tratamiento térmico en sí, no se han demostrado pérdidas de AGPI, mientras que en el caso de los fosfolípidos del tipo plasmalógeno se observó un descenso muy grande (Medina et al., 1993; Aubourg et al., 1995b).

En lo que respecta a las medidas de oxidación, las determinaciones de oxidación primaria (IP; dienos conjugados) y secundaria (i-ATB) demostraron ser poco válidas al objeto de seguir diferencias de calidades en productos enlatados. Se comprobó que el empleo de medios de cobertura conteniendo antioxidantes naturales (aceite de oliva extravirgen) llevaba a productos enlatados con menor grado de oxidación (Medina et al., 1998).

Sobre la base de que los productos de oxidación estén reaccionando con otros constituyentes del pescado, se realizó la detección de dichos compuestos de interacción a través de sus propiedades fluorescentes obteniéndose una buena posibilidad para determinar el grado de alteración de un producto enlatado y la calidad del material de partida empleado en su fabricación (Aubourg y Medina, 1997; Aubourg, 1998).

Uno de los principales problemas asociados con los productos enlatados es el que se refiere a la autenticidad de las muestras empleadas. Este problema es especialmente importante en el caso de los túnidos, por presentar las distintas especies valores comerciales muy diferentes. Recientemente, se han diferenciado satisfactoriamente tres especies de túnidos (albacora, Thunnus alalunga; bonito, Sarda sarda; patudo, Thunnus obesus) a través de parámetros relacionados con la composición fosfolipídica del músculo (Medina et al., 1997).

\subsection{Salazón y secado}

La actividad de agua $\left(a_{w}\right)$ es un factor decisivo de cara a mantener la estabilidad de los alimentos en general, ya que de su valor (entre 0 y 1 , por definición) depende el grado en que se manifiesten las distintas vías de alteración. De acuerdo con la Figura 2 resulta evidente que aquellos alimentos con $a_{w}$ ele- vada, entre los que se encuentran los marinos $\left(a_{w}\right.$ : 0'91-0'97), son altamente perecederos.

En este subapartado se tratarán métodos de conservación que se basan en reducir el valor $a_{w}$. Esto puede llevarse a cabo extrayendo físicamente el agua (proceso de secado), o disminuyendo la cantidad de agua libre o activa a través de la adición de substratos como sal (proceso de salazón), azúcar, propilenglicol, etc. Sin embargo, y de acuerdo con la Figura 2, la oxidación lipídica no se reduce ostensiblemente ni siquiera en el mejor de los casos $\left(a_{w}\right.$ : 0’2-0'3), por lo que será la vía de alteración más importante que puede actuar sobre la calidad del producto final.

Durante el secado se ha observado que la oxidación lipídica medida como i-ATB (Mangaban y Consolación, 1986) o a través del contenido en glicéridos, IY, AGL, IP y contenido en AGPI (Krishnoji Rao y Bandyopadhyay, 1983) resultaba ser una medida fiable del grado de alteración del producto.

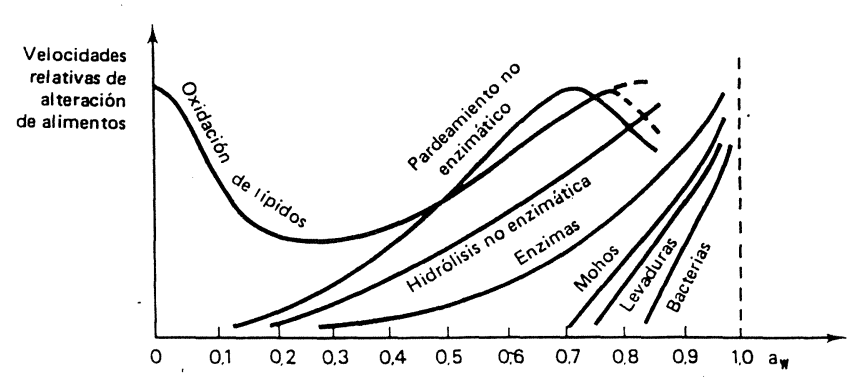

Figura 2

Velocidades relativas de las distintas vías de alteración en función de la actividad de agua *.

* Labuza, 1975.

Durante el procesamiento de anchoado se ha usado el estudio de la formación de compuestos volátiles resultantes de la oxidación lipídica para monitorizar el avance del proceso (Triqui y Reineccius, 1995; Schubring y Oehlenschläger, 1997). Asimismo se ha reconocido como índice aceptable de alteración la formación de AGL (Roldán et al., 1985) o el contenido en fosfolípidos (de Koning et al., 1990).

Es una práctica relativamente habitual combinar salazón y secado (Madrid et al., 1994). En este tipo de experiencias, las medidas tradicionales de IP e iATB (oxidaciones primaria y secundaria, respectivamente) no proporcionaron buenas relaciones con el grado de alteración. Sin embargo, la medida de consumo de oxígeno (Davis et al., 1995) y la detección por fluorescencia de los compuestos de interacción (Maruf et al., 1990; Lubis y Buckle, 1990) proporcionaron las mejores correlaciones con la determina- 
ción organoléptica y el grado de alteración; como resultado de la formación de compuestos de interacción se observó un descenso en el contenido de aminoácidos del tipo lisina, arginina e histidina (Maruf et al., 1990; Smith et al., 1990; Surono et al., 1994). Asimismo, la cuantificación de protones olefínicos respecto a los alifáticos a través de la $\mathrm{RMN}-{ }^{1} \mathrm{H}$ proporcionó un método rápido y útil para determinar el grado de alteración (Saito y Udagawa, 1992).

\subsection{Ahumado}

A menudo el salazón y el secado son seguidos por un proceso de ahumado (Madrid et al., 1994). En este proceso se dota al producto de un nuevo constituyente con características bactericidas y antioxidantes, que provoca asimismo una apariencia, olor y sabor característicos del producto.

La oxidación lipídica, desnaturalización proteica y reacciones de pardeamiento no enzimático pueden ser las principales causas de cambios en la calidad nutritiva de este tipo de productos (Bligh et al., 1988). El empleo de temperaturas elevadas provocará un mayor desarrollo de la oxidación lipídica. Sin embargo, se ha detectado un efecto preservador del ahumado sobre la fracción lipídica en productos de la pesca, basado en la aportación mediante el humo de la madera de sustancias fenólicas con propiedades antioxidantes (Fretheim et al., 1980). Por otra parte, el grado de humedad del producto parece importante; a bajos niveles parece existir una relación directa entre la temperatura del proceso y la velocidad de oxidación del producto ahumado, mientras que con humedades mayores la relación se invierte (Bligh et al., 1988).

Debido a entrar en juego distintos efectos contrapuestos, se obtienen resultados contradictorios respecto a los cambios experimentados por la fracción lipídica y su influencia sobre la calidad del producto final, así como a la posibilidad de emplear algún índice lipídico como medida sensible de calidad.

Bhuiyan et al., (1986) no encontraron diferencias en el contenido de los grupos lipídicos mayoritarios (triglicéridos y fosfolípidos), ni tampoco a nivel de ácidos grasos constituyentes. Sin embargo, otro grupo de experiencias pone de manifiesto que durante el ahumado se producen cambios que pueden tener incidencia en la calidad del producto final. Así, se encontraron incrementos en IP, i-ATB y contenido en AGL (Takiguchi, 1988) y descensos en AGPI (Beltrán y Moral, 1989).

\section{CONSIDERACIONES FINALES}

El objetivo fundamental de un proceso es ofrecer un producto sano que mantenga el valor nutritivo ini- cial. De lo expuesto se observa cómo cada tipo de proceso tiene sus limitaciones particulares, y que una vez controlada la acción microbiana, las alteraciones de la fracción lipídica pueden convertirse en factor limitante a la hora de decidir la aceptabilidad de un producto.

Como resultado de este papel destacado, la determinación de aquellos índices de calidad basados en los cambios de la fracción lipídica pueden proporcionar medidas susceptibles de ser empleadas como parámetros de calidad. Sin embargo, muchos de éstos han encontrado limitaciones al ser aplicados en especies biológicas ricas en AGPI como las marinas y procesos donde se produce un gran avance de la alteración lipídica. En tales casos las medidas de oxidación primaria y secundaria no resultarían útiles, teniendo que recurrir a la medida de los compuestos de interacción producidos por reacción con otros constituyentes de pescado. Como consecuencia, es recomendable el empleo de más de un método al objeto de medir el grado de oxidación lipídica de una muestra.

Dada la incidencia de los cambios de la fracción lipídica sobre el estado de calidad de un producto, se plantea como necesario: a) un conocimiento más profundo de los mecanismos de la hidrólisis y oxidación lipídicas y posterior interacción con otros constituyentes; b) una aplicación correcta de todos aquellos parámetros lipídicos que sean indicadores de degradación o de interacción con otros constituyentes; y c) el estudio de aditivos (antioxidantes naturales y sintéticos, etc.) y la búsqueda de nuevos procesos (o tradicionales modificados) que se centren en inhibir el desencadenamiento de las reacciones degradativas de los lípidos.

\section{AGRADECIMIENTOS}

A la CAICYT (PR 84-0043), CICYT (ALI 88-0145C02-02; ALI 90-0773) y Xunta de Galicia (XUGA 402 01B93) por la ayuda financiera otorgada.

\section{BIBLIOGRAFÍA}

Ababouch, L., Souibri, L., Rhaliby, K., Ouahdi, O., Battal, M. y Busta, F. (1996).— «Quality changes in sardines (Sardina pilchardus) stored in ice and at ambient temperature».-Food Microb. 13, 123-132.

Ackman, R. (1989)._- «atty acids» en "Marine biogenic lipids, fats and oils".-Vol. 1, pp. 103-137, R. Ackman (Ed), CRC Press, Boca Raton, Florida (USA).

Aubourg, S. (1998).- - Lipid changes during long-term storage of canned tuna (Thunnus alalunga)".- $Z$. Lebensm. Unters. Forsch. 206, 33-37.

Aubourg, S., Gallardo, J. y Medina, I. (1997b).— «Changes in lipids during different sterilising conditions of albacore tuna (Thunnus alalunga) canning".--Int. J. Food Sci. Technol. 32, 427-432. 
Aubourg, S. y Medina, I. (1997).-«Quality differences assessment in canned sardine (Sardina pilchardus) by fluorescence detection".-J. Agric. Food Chem. 45, 3617-3621.

Aubourg, S., Medina, I., Gallardo, J. y Pérez-Martín, R. (1995b). - «Efecto del enlatado en aceite y salmuera y su posterior almacenamiento sobre los lípidos de la bacoreta (Euthynnus alletteratus)".-Grasas y Aceites 46, 77-84.

Aubourg, S., Medina, I. y Pérez-Martín, R. (1995a).— «A comparison between conventional and fluorescence detection methods of cooking-induced damage to tuna fish lipids". - Z Lebensm. Unters. Forsch. 200, 252-255.

Aubourg, S., Pérez-Martín, R. y Gallardo, J. (1989).«Stability of frozen albacore (Thunnus alalunga) during steam cooking".- Int. J. Food Sci. Technol. 24, 341-345.

Aubourg, S., Sotelo, C. y Gallardo, J. (1990)._- «Changes in flesh lipids and fill oils of albacore (Thunnus alalunga) during canning and storage».-J. Agric. Food Chem. 38, 809-812.

Aubourg, S., Sotelo, C. y Gallardo, J. (1997a).-«Quality assessment of sardines during storage by measurement of fluorescent compounds".-J. Food Sci. 62, 295-299.

Aubourg, S., Sotelo, C. y Pérez-Martín, R. (1998)."Assessment of quality changes in frozen sardine (Sardina pilchardus) by fluorescence detection".-J. Amer. Oil Chem. Soc. Aceptado, en prensa.

Barassi, C., Pécora, R., Roldán, H. y Trucco, R. (1987)."Total, non-volatile free fatty acids as a freshness index for hake (Merluccius hubbsi) stored in ice».-J. Sci. Food Agric. 38, 373-377.

Beltrán, A. y Moral A. (1989)._- «Effect of smoking on lipid stability in sardine (Sardina pilchardus W.)».-Z. Lebensm. Unters. Forsch. 189, 317-321.

Bhuiyan, A., Ratnayake, W. y Ackamn, R. (1986)."Stability of lipids and polyunsaturated fatty acids during smoking of Atlantic mackerel (Scomber scombrus)". JJ. Am. Oil Chem. Soc. 63, 324-328.

Bligh, E., Shaw, S. y Woyewoda, A. (1988).- - The effect of drying and smoking on lipids of fish" en "Fish smoking and drying", pp. 41-52, J. Burt (Ed).Elsevier App. Sci., Londres y Nueva York.

Careche, M. y Tejada, M. (1991)._«Effect of added lipids on the texture of minced hake (Merluccius merluccius L.), megrim (Lepidorhombus whiffiagonis W.) and sardine (Sardina pilchardus W.) during frozen storage".-Z. Lebensm. Unters. Forsch. 193, 533-537.

Cheftel, J. y Cheftel, H. (1976)._«Introducción a la Biología y Tecnología de alimentos».-Editorial Acribia, Zaragoza.

Davies, L., Smith, G. y Hole, M. (1995).- - Lipid oxidation in salted-dried fish: II. The effect of photosensitisers on the rate of oxidation of a fish oil».-J. Sci. Food Agric. 67, 493-499.

De Koning, A. y Mol, T. (1991).— - Quantitative quality tests for frozen fish: soluble protein and free fatty acid content as quality criteria for hake (Merluccius merluccius) stored at $-18^{\circ} \mathrm{C} »$. - J. Sci. Food Agric. 54, 449-458.

De Koning, A., Mol, T., Przybylak, P. y Thornton, S. (1990). - «The free fatty acid content of fish oil, Part II: The effect of anchovy quality on the free fatty acid content of the resulting anchovy oil and meal».-Fat Sci. Technol. 5, 193-197.
Fernández-Reiriz, M., Pastoriza, L., Sampedro, G. y Herrera, J. (1995). - "Changes in lipids of whole and minced rayfish (Raja clavata) muscle during frozen storage».-Z. Lebensm. Unters. Forsch. 200, 420-424.

Frankel, E. (1991).-— «eview: Recent advances in lipid oxidation".-J. Sci Food Agric. 54, 495-511.

Fretheim, K., Granum, P. y Vold, E. (1980)._- Influence of generation temperature on the chemical composition, antioxidative and antimicrobial effects of wood smoke".-J. Food Sci. 45, 999-1002, 1007.

Gallardo, J., Aubourg, S. y Pérez-Martín, R. (1989)."Lipid classes and their fatty acids at different loci of albacore (Thunnus alalunga): Effects of precooking".J. Agric. Food Chem. 37, 1060-1064.

García-Arias, T., Castrillón, A. y Navarro, P. (1991).«Modificaciones en la grasa del atún blanco (Thunnus alalunga) debidas a la fabricación y almacenamiento de su conserva».-Grasas y Aceites 42, 179-186.

German B., Zhang, H. y Berger, R. (1992).- - Role of lipoxygenases in lipid oxidation in foods" en "Lipid oxidation in food», pp. 74-92, A. St. Angelo (Ed.), American Chemical Society, Washington, DC, USA.

Hale, M. y Brown, T. (1983).— «Fatty acids and lipid classes of three underutilized species and change due to canning".-Marine Fish. Rev. 45, 4-6.

Harris, P. y Tall, J. (1994).- «Rancidity in fish» en «Rancidity in foods".-J. Allen y R. Hamilton (Eds.). Chapman y Hall, Londres (G. B.), pp. 256-272.

Hearn, T., Sgoutas, S., Sgoutas, D. y Hearn, J. (1987).«Polyunsaturated fatty acids and fat in fish flesh for selecting species for health benefits".-J. Food Sci. 52, 1209-1211.

Hidalgo, J., Zamora, R. y Girón, J. (1992).-—Modificaciones producidas en las proteínas alimentarias por su interacción con lípidos peroxidados. III. Consecuencias nutricionales y toxicológicas".-Grasas y Aceites 43, 97-100.

Joly, A., Huidobro, A. y Tejada, M. (1997)._- Influence of lipids on dimethylamine formation in model systems of hake (Merluccius merluccius) kidney during frozen storage".-Z. Lebensm. Unters. Forsch. 205, 14-18.

Karaçam, H. y Boran, M. (1996).- «Quality changes in frozen whole and gutted anchovies during storage at $-18^{\circ} \mathrm{C} »$. - Int. J. Food Sci. Technol. 31, 527-531.

Kinsella, J. (1987).-— «Dietary fats and cardiovascular disease» en "Seafoods and Fish Oils in Human Health and Disease".-R. Lees, y M. Karel (Eds.), pp. 1-23, Marcel Dekker, Inc., New York and Basel.

Krishnoji Rao, B. y Bandyopadhyay, C. (1983).-_Lipid composition of salted sun-dried Indian mackerel (Rastrelliger kanagurta)».-J. Food Sci. Technol. 20, 62-64.

Labuza, T. (1975).- -Sorption phenomena in foods: Theoretical and practical aspects" en "Theory, determination and control of physical properties of food materials" p. 197.-C. Rha (Ed.), Reidel, Dordrecht, Netherlands.

Linko, Y. y Hayakawa, K. (1996).- «Docosahexaenoic acid: A valuable nutraceutical?".-Trends Food Sci. Technol. 7, 59-63.

Lubis, Z. y Buckle, K. (1990).-«Rancidity and lipid oxidation of dried-salted sardines".-Int. J. Food Sci. Technol. 25, 295-303.

Mackie, I. (1993).- «The effects of freezing on flesh proteins".-Food Rev. Internat. 9, 575-610.

Madrid, A., Madrid, J. y Madrid, R. (1994).—-Tecnología del pescado y productos derivados" p. 56.-A. Madrid Vicente, Ediciones y Mundi-Prensa Libros, S. A., España. 
Mangaban, M. y Consolación, F. (1986).-—Storage stability of dried fish: lipid oxidation and microbial growth at different relative humidity levels".-Phil. Agr. 69, 25-32.

Maruf, F., Ledward, D., Neale, R. y Poulter, R. (1990)."Chemical and nutritional quality of Indonesian dried-salted mackerel (Rastrelliger kanagurta)».-Int. J. Food Sci. Technol. 25, 66-77.

Medina, I., Aubourg, S. y Pérez-Martín, R. (1993)."Analysis of 1-O-alk-1-enylglycerophospholipids of albacore tuna (Thunnus alalunga) and their alterations during thermal processing".-J. Agric. Food Chem. 41, 2395-2399.

Medina, I., Aubourg, S. y Pérez-Martín, R. (1997)."Species differentiation by multivariate analysis of phospholipids from canned Atlantic tuna».-J. Agric. Food Chem. 45, 2495-2499.

Medina, I., Sacchi, R. y Aubourg, S. (1994).- $-{ }^{13}$ C-NMR monitoring of FFA release after fish thermal processing".-J. Amer. Oil Chem. Soc. 71, 479-482.

Medina, I., Sacchi, R. y Aubourg, S. (1995).-«A ${ }^{3} \mathrm{C}-\mathrm{NMR}$ study of lipid alterations during fish canning: Effect of filling medium".-J. Sci. Food Agric. 69, 445-450.

Medina, I., Sacchi, R., Biondi, L., Aubourg, S. y Paolillo, L. (1998).- - Effect of packing media on the oxidation of canned tuna lipids. Antioxidant effectiveness of extra virgin olive oil».-J. Agric. Food Chem. 46, 1150-1157.

Mielche, M. y. Bertelsen, G. (1994).— «Approaches to the prevention of warmed-over flavour". - Trends Food Sci. Technol. 5, 322-327.

Mohri, S., Cho, S.-Y., Endo, Y. y Fujimoto, K. (1992)."Linoleate 13(S)-lipoxygenase in sardine skin».-J. Agric. Food Chem. 40, 573-576.

Navarro, P. (1991)._ «Valor nutritivo del pescado. I. Pescado fresco».-Rev. Agroq. Tecn. Alim. 31, 330-342.

Nielsen, H., Löliger, J. y Hurrell, R. (1985)._- «Reactions of proteins with oxidising lipids. 1. Analytical measurements of lipid oxidation and of amino acid losses in a whey protein-methyl linolenate model system.»-Brit. J. Nutrit. 53, 61-73.

Nunes, M., Batista, I. y Morao de Campos, R. (1992).«Physical, chemical and sensory analysis of sardine (Sardina pilchardus) stored in ice".-J. Sci. Food Agric. 59, 37-43.

Ölafsdóttir, G., Martinsdóttir, E., Oehlenschläger, J., Dalgaard, P., Jensen, B., Undeland, I., Mackie, I., Henehan, G., Nielsen, J. y Nilsen, H. (1997). — «Methods to evaluate fish freshness in research and industry".-Trends Food Sci. Technol. 8, 258-265.

Pérez-Villarreal, B. y Howgate, P. (1991).- «Deterioration of European hake (Merluccius merluccius) during frozen storage".-J. Sci. Food Agric. 55, 455-469.

Pigott, G. y Tucker, B. (1987).- «Science opens new horizons for marine lipids in human nutrition".-Food Rev. Intern. 3, 105-138.

Pokorný, J. (1981).— «Browning from lipid-protein interactions".-Prog. Food Nutr. Sci. 5, 421-428.

Rehbein, H. y Orlick, B. (1990).- "Comparison of the contribution of formaldehyde and lipid oxidation products to protein denaturation and texture deterioration during frozen storage of minced ice-fish fillet (Champsocephalus gunnari $y$ Pseudochaenichthys georgianus)".- Int. J. Refrig. 13, 336-341.

Roldán, H., Barassi, C. y Trucco, R. (1985).— «Increase on free fatty acids during ripening of anchovies (Engraulis anchoíta)». - J. Food Technol. 20, 581-585.

Saito, H. y Udagawa, M. (1992).—《Assessment of oxidative deterioration of salted dried fish by Nuclear Magnetic Resonance».-J. Amer. Oil Chem. Soc. 69, 1157-1159.

Schellekens, M. (1996).- «New research issues in sous-vide cooking".-Trends Food Sci. Technol. 7, 256-262.

Schubring, R. y Oehlenschläger, J. (1997)._«Comparison of the ripening process in salted Baltic and North Sea herring as measured by instrumental and sensory methods".-Z. Lebensm. Unters. Forsch. 205, 89-92.

Smith, G., Hole, M. y Hanson, S. (1990).— «Assessment of lipid oxidation in Indonesian salted-dried Marine catfish (Arius thalassinus)».-J. Sci. Food Agric. 51, 193-205.

Simopoulos, A. (1997).- «Nutritional aspects of fish» en "Seafood from producer to consumer, integrated approach to quality».-J. Luten, T. BØrresen y J. Oehlenschläger (Eds.). Elsevier, Amsterdam (Holanda), pp. 589-607.

Sotelo, C., Piñeiro, C. y Pérez-Martín, R. (1995).«Denaturation of fish proteins during frozen storage: role of formaldehyde».-Z. Lebensm. Unters. Forsch. 200, 14-23.

Surono, K., Anthony Taylor, A. y Smith, G. (1994).- «The effect of different salting procedures and qualities of raw material on some nutrients during processing and storage of salted-dried mackerel».-Int. J. Food Sci. Technol. 29, 179-183.

Suzuki, H., Chung, S., Isobe, S., Hayakawa, S. y Wada, S. (1988). - «Changes in $\omega-3$ polyunsaturated fatty acids in the chum salmon muscle during spawning migration and extrusion cooking".-J. Food Sci. 53, 1659-1661.

Takiguchi, A. (1988). - «Effects of smoking on lipid oxidation in niboshi and niboshi powder".-Bull. Jap. Soc. Scient. Fish. 54, 869-874.

Tóth-Markus, M. y Sass-Kiss, A. (1993).— «Effect of cooking on the fatty acid composition of silver carp (Hypophtalmichtis molitrix, V.)».-Acta Alimentaria 22, 25-35.

Triqui, R. y Reineccius, G. (1995)._-Changes in flavor profiles with ripening of anchovy (Engraulis encrasicholus)". - J. Agric. Food Chem. 43, 1883-1889.

Verma, J., Srikar, L., Sudhakara, N. y Sarma, J. (1995).«Effects of frozen storage on lipid freshness parameters and some functional properties of oil sardine (Sardinella longiceps) mince».-Food Res. Intern. 28, 87-90. 